\title{
Índice HOMA (homeostasis model assessment) na prática clínica: uma revisão
}

\author{
HOMA (homeostasis model assessment) index in clinical practice: a review
}

Ernesto Pereira de Oliveira'; Mirabeau Levi Alves de Souza ${ }^{2}$; Maria das Dores Acioli de Lima ${ }^{3}$

\section{unitermos resumo}

Resistência à insulina

Introdução: Progressivamente, desde a década de 1980, a resistência à insulina vem sendo associada Sindrome $X$ metabólica ao risco de doenças cardiovasculares e diabetes mellitus tipo 2. Um dos métodos mais empregados para Diagnóstico sua estimativa é o índice HOMA que, embora bem estabelecido para estudos epidemiológicos, ainda carece de resultados consensuais para aplicação mais consistente na prática clínica, campo em que HOMA ampliaria a possibilidade de antecipação de medidas preventivas. Método: Conduzimos uma revisão sistemática nesse campo, considerando o período 2000-2004. Resultados: No período, a sigla HOMA aparece em 670 artigos, dos quais 49 foram selecionados pela ênfase na utilização na prática clínica. A maioria traz o índice como medida de desfecho em ensaios terapêuticos e são poucos aqueles com desenho mais apropriado à avaliação de testes de diagnóstico. Discussão: $\mathrm{O}$ índice HOMA apresenta uma variabilidade que reduz a sensibilidade e a especificidade do método, assim como o poder discriminatório entre resultados, o que limita seu uso na prática clínica, mas não impede sua aplicação em estudos epidemiológicos de base populacional. Conclusão: Os resultados apontam para a necessidade de uma padronização das condições de realização do teste, a definição de um valor de corte mais amplamente aplicável e critérios para interpretação e utilização dos resultados.
\end{abstract}

abstract

Introduction: Since the 1980's, insulin resistance (IR) has been associated to the risk of cardiovascular disease and type 2 diabetes mellitus. HOMA index is one of the methods used for IR measurement, mainly in epidemiological studies. However, the current state of application of this index in clinical practice, where it should be helpful in the anticipation of preventive intervention, still needs to achieve consensual results. Method: A systematic review of the literature, from 2000 to 2004, relating to HOMA index in clinical practice. Results: Among 670 articles where the term HOMA was found, 49 met eligibility criteria. Most of them refer to the index as an outcome measure in therapeutic trials and only a few are designed to evaluate diagnosis tests. Discussion: HOMA index variability reduces the sensitivity and the specificity of the method as much as the discriminant power of the results, which turn to be a limitation for clinical trials. However, it does not prevent the index to be reliably used in population-based epidemiological investigation. Conclusion: The results point out the need of establishment of standard conditions to improve the test, better definition of cut-off limits as much as confident criteria for the HOMA index interpretation. key words

Insulin resistance

Metabolic syndrome X

Diagnosis

HOMA 


\section{Introdução}

Métodos para determinação da resistência à insulina (RI) e da capacidade funcional das células beta $(\mathrm{BcC})$ têm se multiplicado a partir de Olefsky et al. ${ }^{(61)}$, Insel et al.(32), Bergman et al. ${ }^{(8)}$, entre outros, e podem ser agrupados em dois modelos: um, baseado na medida dinâmica das respostas insulínica e/ou glicêmica após estímulo, tais como clamp euglicêmico ${ }^{(15)}$, modelo mínimo ${ }^{(8)}$, continuous infusion of glucose with model assessment (CIGMA) ${ }^{(30)} \mathrm{e}$ insulin sensitivity index (ISI)(55); o outro, assentado em medidas estáticas, ou instantâneas, de um ou mais constituintes plasmáticos, mais freqüentemente insulina, glicose, pró-insulina e peptídeo C, representado pelos índices homeostasis model assessment $(\mathrm{HOMA})^{(7)}$, e quantitative insulin-sensitivity check index (QUICKI) ${ }^{(40)}$.

Tais técnicas vêm servindo ao entendimento dos mecanismos regulatórios envolvidos na homeostase da glicose e na patogênese do diabetes tipo $2^{(46,56,86)}$, à avaliação de risco e predição do desenvolvimento de diabetes tipo 2 (DM2) em estudos epidemiológicos ${ }^{(23,26)}$, para avaliação do curso clinicoterapêutico do diabetes ${ }^{(39,76,82)}$, para medida da RI associada à síndrome dos ovários policísticos ${ }^{(3,4,83,84) ;}$ bem como para a determinação da Rl e da $\mathrm{BcC}$, base para trabalhos acerca da patogenia genético-molecular da RI e dos distúrbios da tolerância à glicose $\mathrm{e}^{(7,34)}$.

As abordagens pertinentes ao primeiro modelo são mais apropriadas aos estudos da fisiopatologia da homeostase da glicose e, em tese, mais sensíveis, por medirem efetivamente tanto a sensibilidade hepática quanto a dos tecidos periféricos à insulina, revelando com mais força eventos que pré-existem à perda da homeostase da glicose, portanto, mais precoces no curso da patogênese do DM2, como a perda do pico primário de resposta insulínica por decadência do potencial de reserva celular do hormônio e o aumento da relação pró-insulinemia/insulinemia, sob estímulo insulinogênico( ${ }^{(21,79)}$. Contudo, são mais invasivas, trabalhosas e dispendiosas, o que dificulta suas aceitabilidade e aplicabilidade, sobretudo em estudos de base populacional(51,71,78).

Os métodos inspirados no segundo modelo medem basicamente a sensibilidade hepática à insulina, estimam a sensibilidade periférica em função dela e equacionam dados do equilíbrio insulina/glicose obtidos a partir de testes dinâmicos pós-estímulo, clamps e modelos mínimos, para inferir medidas dos níveis de $\mathrm{RI}$ e de $\mathrm{BcC}$. Por serem de realização mais simples, melhoram os aspectos de aceitabilidade e aplicabilidade ${ }^{(55,58,71)}$. Tais sistemas, mais especificamente o índice HOMA, têm sido validados diante do padrão-ouro, o clamp euglicêmico hiperinsulinêmico (CEH), por vários autores, dando sustentação ao seu uso principalmente em estudos epidemiológicos ${ }^{(11,22,31,39,70,85)}$.

A medida da RI e da BcC ganhou maior interesse a partir dos trabalhos de Reaven, que em 1988 denominou de síndrome de resistência à insulina a coexistência freqüente de hipertensão, DM2, dislipidemia e obesidade. Desde então, passou-se a aventar a relação direta entre RI e risco cardiovascular, mesmo em indivíduos ainda sem alterações da tolerância à glicose $\mathrm{e}^{(5,14,78)}$.

$\mathrm{O}$ índice HOMA, descrito por Matthews et al. ${ }^{(58)}$, pode ser calculado de duas maneiras: a estimativa da RI, na qual HOMA-RI = insulinemia de jejum ( $\mathrm{mU} / \mathrm{L}) \times$ glicemia de jejum ( $\mathrm{mmol} / \mathrm{L}) / 22,5$; e a determinação da $\mathrm{BcC}$, onde HOMA-BcC $=(20 \mathrm{x}$ insulinemia de jejum $[\mathrm{mU} / \mathrm{L}]) /($ glicemia de jejum $[\mathrm{mmol} / \mathrm{L}]-3,5)$. Por ser estimado simplesmente a partir da determinação da insulinemia e da glicemia de jejum, vem sendo amplamente utilizado e referido na literatura, sobretudo em estudos populacionais $(1,12,18,20,22,75,77)$.

Interessados na utilização do índice HOMA enquanto instrumento de diagnóstico laboratorial de RI, o que permitiria aplicação de medidas preventivas diante do risco de doença cardiovascular (DCV) e DM2, ressentimo-nos da ausência de trabalhos que validem sua aplicação na prática clínica, ainda que esta seja referida por alguns autores ${ }^{(9,31,}$ 39, 499. Assim, decidimos realizar esta revisão sistemática da literatura para avaliar como, de fato, está sendo posta tal aplicabilidade.

\section{Método}

Levantamento bibliográfico foi realizado nas bases Medline e Lilacs, através da interface de recuperação da Biblioteca Virtual em Saúde, a partir do ano 2000.

Leituras iniciais de artigos encontrados através dos Descritores em Ciências da Saúde (DeCS) sindrome $X$ de resistência à insulina e resistência à insulina permitiram definir os termos para buscas mais direcionadas: HOMA, diet, exercise, physical, responders, insulin-sensitizing, sulphonylurea, thiazolidinediones, troglitazone, therapy, clinical practice, variability, variation, reproductibility, reference values, roc curve, os DeCS citados, acompanhados ou não do aspecto diagnóstico, além do DeCS hipoglycemic agents. As expressões de pesquisa foram construídas combinando esses termos, mantendo constante a sigla HOMA, buscada como palavra em qualquer campo pesquisado. 
Os artigos tratados efetivamente nesta revisão foram selecionados quando a leitura dos resumos revelou possibilidade de aplicação do índice HOMA na clínica em acompanhamento terapêutico, diagnóstico ou prognóstico, em ensaios clínicos, em estudos longitudinais e em desenhos específicos para avaliação do método, validação e definição de valores de corte.

\section{Resultados}

Quando buscamos a ocorrência da sigla HOMA nas bases de dados, nos últimos cinco anos, encontramos vinte publicações na LILACS e 650 na MEDLINE.

Quarenta e nove artigos foram selecionados, segundo os critérios pré-definidos. Eles analisam e referem os seguintes aspectos relacionados à aplicação clínica do índice HOMA: 1) avaliação do risco de desenvolvimento de diabetes; 2 ) avaliação do risco de doença cardiovascular em diabéticos e não-diabéticos; 3) seguimento terapêutico do DM2, inclusive diagnóstico da fase de requerimento de insulina; e 4) acompanhamento do tratamento da síndrome de ovários policísticos (SOP) com tiazolidinedionas.

A maioria dos trabalhos selecionados (49\%) são ensaios terapêuticos que usam o HOMA como uma das medidas

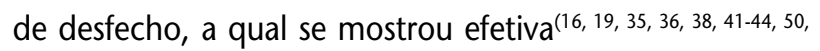
52-54, 63, 66-69, 72-74,80). Cinco outros artigos (10\%) apresentaram desenhos mais apropriados ao estudo de testes de diagnóstico e concluíram que o HOMA, sobretudo o HOMA-BcC, é útil ao acompanhamento terapêutico do DM2, à predição da falência terapêutica do uso de sulfoniluréia e à escolha terapêutica, inclusive entre metformina e pioglitazona ${ }^{(9,}$ $28,39,59,76)$.

A predição do DM2 foi contemplada por um artigo (2\%) que, em concordância com Haffner et al. ${ }^{(22)}$, conclui pela capacidade do HOMA-RI em predizer o desenvolvimento de $\mathrm{DM}^{(65)}$ e se contrapõe ao achado de Yoshinaga et al. ${ }^{\left({ }^{(8)}\right)}$, que relatam a ausência desse poder preditivo. A falta de consenso pode estar influenciada por diferenças étnicas na fisiopatologia do DM2, bem como de faixa etária e composição corporal dos indivíduos envolvidos nas pesquisas.

O risco de desenvolvimento de $D C V$ em não-diabéticos foi abordado por quatro artigos (8\%): um deles conclui que alterações aterogênicas em pré-diabéticos são principalmente vistas em indivíduos cujo HOMA-Rl indica R( ${ }^{(24)}$; Hanley et al. ${ }^{(25)}$ demonstram associação significante entre HOMA-RI e risco de DCV; para um terceiro relato, HOMA-RI não prediz
DCV independentemente de outros fatores de risco(6); ${ }^{(65}$ o quarto não recomenda o HOMA-RI como índice de RI em idosos, pela ausência de correlação do HOMA-RI com o CEH, dado que se atribui à possibilidade de tolerância prejudicada à glicose nesses pacientes ${ }^{(17)}$.

Bonora et al. ${ }^{(10)}$, estudando 1.326 pacientes com DM2, concluem que HOMA-RI é preditor independente de DCV; Hirose et al. ${ }^{(27)}$ relatam que RI, definida por HOMA-RI, prediz hipertensão arterial em japoneses de meia-idade; Adachi et al. ${ }^{(2)}$ se referem especificamente à relação entre RI e acidente vascular cerebral (AVC) e demarcam que HOMA-RI não prediz o evento em diabéticos japoneses. Todos esses achados estão associados ao risco de DCV.

Quatro artigos (8\%) tratam de abordagens relativas à definição de valores de referência para o índice HOMA; o mais recente envolve 6.511 americanos $^{(1,13,46,75)}$.

Os demais relatos avaliam eficácia terapêutica da SOP com derivados de tiazolidinedionas ${ }^{(6,87,88)}$, além dos cinco trabalhos (10\%) direcionados à validação do HOMA.

\section{Discussão}

Quanto ao uso do índice HOMA na prática clínica, a análise dos artigos revela conclusões que se contradizem ou que são mais restritas a populações específicas.

Há ausência de mais trabalhos com desenhos específicos à avaliação de testes de diagnóstico, conforme descrevem Newman et al. $\left.{ }^{(60)}: 1\right)$ estudos sobre o efeito do teste no desfecho desejado; 2) trabalhos sobre exeqüibilidade, custos e riscos do teste; 3) avaliações da influência do teste nas decisões clinicoterapêuticas; 4) avaliações sobre exatidão e reprodutibilidade do teste, que permitiriam dirimir questões preliminares, algumas já reconhecidas por Matthews et al. ${ }^{(58)}$.

É perceptível a falta de um valor de corte estabelecido como referência para classificar os resultados dos pacientes. Desde que Matthews et al. ${ }^{(58)}$ definiram que indivíduos hígidos, com menos de 35 anos e peso corporal normal tinham HOMA-RI $=1$ e HOMA-BcC $=100 \%$, a literatura revela variação entre as propostas dos diversos autores para HOMA-RI: 3,0, definido em estudo envolvendo 90 japoneses hígidos e 281 diabéticos do tipo $2^{(47)} ; 2,8 \pm 2,4$, em estudo com 6.511 americanos $^{(13)} ; 1,96 \pm 0,57$, para chilenos não-obesos ${ }^{(1)} ; 2,24 \pm 1,26$, encontrado por Gokcel et al. ${ }^{(20)} \mathrm{em}$ turcos, e 2,5 considerado por Taniguchi et al. ${ }^{(75)}$ em um estudo que avalia a existência de duas variantes de não-obesos levemente diabéticos, com e sem RI. 
Tal variação pode estar assentada nas diferenças populacionais. Além disso, poderá estar influenciada pela falta de padronização das condições para realização das medidas de insulina e glicose que servem ao cálculo do índice HOMA, o que se ilustra pela diversidade de sistemas analíticos utilizados.

Outro elemento importante a ser considerado é a variabilidade biológica (VB) da insulinemia que é devida a fatores como estresse ${ }^{(29)}$, exercício físico ${ }^{(48)}$ e o ritmo pulsátil de secreção insulínica ${ }^{(57)}$. Tais fatores contribuem para que a VB do índice HOMA-RI oscile de 7,1\% em não-diabéti$\cos$ a $24,2 \%$ em diabéticos tipo 2 , segundo Jayagopal ${ }^{(33)}$, ou mesmo $31 \%$ para HOMA-RI e $32 \%$ para HOMA-BcC, conforme Matthews et al. ${ }^{(58)}$.

Tal variabilidade limita o uso clínico do índice e reduz a sensibilidade e a especificidade do método, assim como o poder discriminatório entre resultados consecutivos de um mesmo indivíduo ${ }^{(33)}$, embora tenha pouco impacto nos estudos populacionais.

Uma solução plausível sugerida na literatura, inclusive por Matthews et al. ${ }^{(58)}$, é a coleta de amostras múltiplas, pelo menos três, dentro do período de quatorze minutos em que se completa o ciclo de secreção da insulina. Além disso, as definições de comportamento alimentar, sono/vigília e atividade física do paciente nos dias que precedem o teste podem contribuir para melhorar a reprodutibilidade dos índices.

Outro detalhe importante a ser considerado para o possível sucesso da utilização do HOMA na prática clínica é o grau de especificidade analítica do ensaio para medida da insulina. Níveis de reação cruzada com pró-insulina e outros precursores podem aumentar a variabilidade inter e intra-ensaio, se considerarmos a tendência de indivíduos com mais RI a exibir uma maior fração de pró-insulina circulante que aqueles com menos Rl, o que contribuiria, falsamente, para o achado de elevações da insulinemia. Os ensaios disponíveis têm percentuais de cruzamento que podem variar de $0,2 \%$ a $40 \%$, ou até mais. Os mais específicos têm sido mais recomendados.

Sente-se falta de estudos que demonstrem a efetiva utilidade clínica do teste, influenciando na definição de medidas terapêuticas ou preventivas, em comparação com alternativas mais econômicas, tais como a medida da insulinemia de jejum ${ }^{(23)}$, da pró-insulinemia ${ }^{(37,62)}$ ou do diâmetro da cintura ${ }^{(45)}$ como indicadores de resistência à insulina e preditores de DM2 ou a utilização das corriqueiras medidas de glicemias de jejum e pós-prandial, além da determinação de hemoglobina glicosilada e frutosamina, para acompanhamento terapêutico de DM2 e definição de possível insucesso(66).

\section{Conclusão}

Não obstante a importância do diagnóstico de RI para prevenção de DM2 e DCV, sobretudo, fica clara a necessidade de que a escolha da utilização do índice HOMA passe por uma padronização das condições pré-analíticas e analíticas de realização do teste, pela definição de um valor de corte amplamente aplicável ou, pelo menos, pertinente à população-alvo, bem como pelo estabelecimento de critérios de interpretação que orientem as decisões demandadas dos resultados, com o que também concorda uma ampla revisão sobre os usos do HOMA publicada por Wallace et al. ${ }^{(81)}$.

\section{Referências}

I.ACOSTA,A. M. et al. Determination of the insulin resistance index by the homeostasis model assessment in a population of Metropolitan Region in Chile. Rev Med Chil, v. I30, n. II, p. 1227-31, 2002.

2. ADACHI, $\mathrm{H}$. et al. Is insulin resistance or diabetes mellitus associated with stroke? An 18-year follow-up study. Diabetes Res Clin Pract, v. 5I, n. 3, p. 215-23, 200 I.

3. AMADOR, N. et al. Comparación del HOMA con el modelo mínimo para medir sensibilidad a la insulina en el síndrome de ovarios poliquísticos. Rev Invest Clin, v. 53, n. 5, p.407-12, 2001.

4. AYALA, A. R. et al. Índice de resistencia a la insulina: medición y potencial en reproducción humana. Ginecol Obstet Mex, v.
69, n. 7, p. 268-7I, 200I.

5. BARZILAV, J. I. et al. Cardiovascular disease in older adults with glucose disorders: comparisons of American Diabetes Association of diabetes mellitus with WHO criteria. Lancet, v. 354, p. 622-5, 1999.

6. BELLI, S. H. et al. Effect of rosiglitazone on insulin resistance, growth factors, and reproductive disturbances in women with polycystic ovary syndrome. Fertil Steril, v. 8I, n. 3, p. 624-9, 2004.

7. BERGMAN, R. N. et al. Minimal model-based insulin sensitivity has greater heritability and a different genetic basis than homeostasis model assessment or fasting insulin. Diabetes, v. 52, n. 8, p. 2168-74, 2003. 
8. BERGMAN, R. N. et al. Quantitative estimation of insulin sensitivity. Am J Physiol, v. 236, p. E667-E677, 1979.

9. BERMÚDEZ, P.V. et al. Homeostasis model assessment (HOMA) en pacientes diabéticos tipo 2. Arch Venez Farmacol Ter, v. 19, n. I, p. 53-7, 2000.

10. BONORA, E. et al. HOMA-estimated insulin resistance is an independent predictor of cardiovascular disease in type 2 diabetic subjects: prospective data from the Verona Diabetes Complications Study. Diabetes Care, v. 25, n. 7, p. I |35-4|, 2002.

I I. BONORA, E. et al. Homeostasis model assessment closely mirrors the glucose clamp technique in the assessment of insulin sensitivity: studies in subjects with various degrees of glucose tolerance and insulin sensitivity. Diabetes Care, v. 23, n. I, p. 57-63, 2000.

12. BONORA, E. et al. Population-based incidence rates and risk factors for type 2 diabetes in white individuals: the Bruneck Study. Diabetes, v. 53, n. 7, p. 1782-9, 2004.

13. BRAVATA, D. M. et al.Two measures of insulin sensitivity provided similar information in US population. J Clin Epidemiol, v. 57, n. II, p. 1214-7, 2004.

14. COUTINHO, M. et al. The relationship between glucose and incident cardiovascular events. Diabetes Care, v. 22, p. 23340, 1999.

I5. DE FRONZO, R. A. et al. Glucose clamp technique: a method for quantifying insulin resistance. Am J Physiol, v. 237, p. E2|4-E223, 1979.

16. DUMORTIER, M. et al. Low intensity endurance exercise targeted for lipid oxidation improves body composition and insulin sensitivity in patients with the metabolic syndrome. Diabetes Metab, v. 29, n. 5, p. 509- 18, 2003.

17. FERRARA, C. M. et al. Limited value of the homeostasis model assessment to predict insulin resistance in older men with impaired glucose tolerance. Diabetes Care, v. 24, n. 2, p. 245-9, 2001.

18. FUKUSHIMA, M. et al. Insulin secretion and insulin sensitivity at different stages of glucose tolerance: a cross-sectional study of Japanese type 2 diabetes. Metabolism, v. 53, n. 7, p. 831-5, 2004.

19. FÜLLERT, S. et al. Effects of pioglitazone in nondiabetic patients with arterial hypertension: a double-blind, placebocontrolled study. J Clin Endocrinol Metab, v. 87, n. 12, p. 5503-6, 2002.

20. GOKCEL, A. et al. Detection of insulin resistance in Turkish adults: a hospital-based study. Diabetes Obes Metab, v. 5, n. 2, p. 126-30, 2003.

21. GROSS, J. L. et al. Diabetes melito: diagnóstico, classificação e avaliação do controle glicêmico. Arq Bras Endocrinol Metab, v. 46, n. I, p. 16-26, 2002.

22. HAFFNER, S. M. et al. A prospective analysis of the HOMA model. The Mexico City Diabetes Study. Diabetes Care, v. 19, n. 10, p. II38-41, 1996.

23. HAFFNER, S. M. et al. Decreased insulin secretion and increased insulin resistance are independently related to the 7-year risk of NIDDM in Mexican-Americans. Diabetes, v. 44, n. 12 , p. |386-9|, 1995.

24. HAFFNER, S. M. et al. Insulin-resistant prediabetic subjects have more atherogenic risk factors than insulin-sensitive prediabetic subjects: implications for preventing coronary heart disease during the prediabetic state. Circulation, v. I0 I, n. 9, p. 975-80, 2000.

25. HANLEY, A. J. et al. Homeostasis model assessment of insulin resistance in relation to the incidence of cardiovascular disease: the San Antonio Heart Study. Diabetes Care, v. 25, n. 7, p. 1 177-84, 2002.

26. HANLEY, A. J. et al. Prediction of type 2 diabetes using simple measures of insulin resistance: combined results from San Antonio Heart Study, the Mexico City Diabetes Study and the Insulin Resistance Atherosclerosis Study. Diabetes, v. 52, n. 2, p. 463-9, 2003.

27. HIROSE, H. et al. Insulin resistance and hypertension: seven-year follow-up study in middle-aged Japanese men (the KEIO study). Hypertens Res, v. 26, n. 10, p. 795-800, 2003.

28. HIROSE, T. et al. Characteristics of type 2 diabetic patients responding to voglibose administration as an adjunct to sulfonylurea. Diabetes Res Clin Pract, v. 54, n. I, p. 9- 15, 200 I.

29. HOLMAN, R. R. et al. The basal plasma glucose: a single relevant index of maturity onset diabetes. Clin Endocrinol, v. 14, p. 279-86, 1980

30. HOSKER, J. P. et al. Continuous infusion of glucose with model assessment: measurement of insulin resistance and beta-cell function in man. Diabetologia, v. 28, p. 40 I- I I, 1985.

31. IKEDA, Y. et al. Clinical significance of the insulin resistance index as assessed by homeostasis model assessment. Endocr J, v. 48, n. I, p. $81-6,200$ I.

32. INSEL, P.A. et al. Insulin control of glucose metabolism in man: a new kinetic analysis. J Clin Invest, v. 55, p. 1057-66, 1975.

33. JAYAGOPAL,V. et al. Biological variations of homeostasis model assessment-derived insulin resistance in type 2 diabetes. Diabetes Care, v. 25, n. I I, p. 2022-5, 2002.

34. JENKINS, A. B. et al. Improved indices of insulin resistance and insulin secretion for use in genetic and population studies of type 2 diabetes mellitus. Twin Res, v. 3, n. 3, p. 148-51, 2000.

35. JONES, T. A. et al. Addition of rosiglitazone to metformin is most effective in obese, insulin-resistant patients with type 2 diabetes. Diabetes Obes Metab, v. 5, n. 3, p. 163-70, 2003.

36. JOSSE, R. G. et al. Acarbose in the treatment of elderly patients with type 2 diabetes. Diabetes Res Clin Pract, v. 59, n. I, p. 37-42, 2003.

37. KAHN, S. E. et al. Proinsulin levels predict the development of non-insulin-dependent diabetes mellitus (NIDDM) in Japanese-American men. Diabet Med, v. 13, n. 9 suppl 6, p. S63-6, 1996.

38. $\mathrm{KATOH}$, S. et al.Troglitazone prevents the rise in visceral adiposity and improves fatty liver associated with sulfonylurea therapy: a randomized controlled trial. Metabolism, v. 50, n. 4, p. 4|4-7, 200।.

39. KATSUKI, A. et al. Homeostasis model assessment is a reliable indicator of insulin resistance during follow-up of patients with type 2 diabetes. Diabetes Care, v. 24, n. 2, p. 362-5, 2001.

40. KATZ, A. et al. Quantitative insulin sensitivity check index: a simple, accurate method for assessing insulin sensitivity in 
humans.J Clin Endocrinol Metab, v. 85, p. 2402-10, 2000.

4I. KAWANO, Y. et al. Effects of a low-energy diet and an insulin-sensitizing agent on ambulatory blood pressure in overweight hypertensive patients. J Hypertens, v. 18, n. 10, p. $|45|-5,2000$.

42. KERENYI, Z. et al. Combination therapy with rosiglitazone and glibenclamide compared with upward titration of glibenclamide alone in patients with type 2 diabetes mellitus. Diabetes Res Clin Pract, v. 63, n. 3, p. 21 3-23, 2004.

43. KINOSHITA, M. et al. Combination therapy of exercise and angiotensin-converting enzyme inhibitor markedly improves insulin sensitivities in hypertensive patients with insulin resistance. Circ J, v. 66, n. 7, p. 655-8, 2002.

44. KISHIMOTO, H. et al. Effect of short-term low-intensity exercise on insulin sensitivity, insulin secretion, and glucose and lipid metabolism in non-obese Japanese type 2 diabetic patients. Horm Metab Res, v. 34, n. I, p. 27-31, 2002.

45. KRIKETOS,A. D. et al. Central fat predicts deterioration of insulin secretion index and fasting glycaemia: 6-year follow-up of subjects at varying risk of type 2 diabetes mellitus. Diabet Med, v. 20, n. 4, p. 294-300, 2003.

46. KUROE,A. et al. Impaired beta-cell function and insulin sensitivity in Japanese subjects with normal glucose tolerance. Diabetes Res Clin Pract, v. 59, n. I, p. 7I-7, 2003.

47. KUWANA B. et al. Reference value and cut-off value for diagnosis of insulin resistance in type 2 diabetes mellitus [resumo]. Rinsho Byori, v. 50, n. 4, p. 398-403, 2002.

48. LEBLANC, J. et al. Daily variations of plasma glucose and insulin in physically trained and sedentary subjects. Metabolism, v. 32, p. 552-7, 1983.

49. LEGRO, R. S. et al. Detecting insulin resistance in polycystic ovary syndrome: purposes e pitfalls. Obstetrical and Gynecological Survey, v. 59, n. 2, p. I4|-54, 2004.

50. LIN, S. H. et al. Rosiglitazone improves glucose metabolism in nondiabetic uremic patients on CAPD. Am J Kidney Dis, v. 42, n. 4, p. 774-80, 2003.

5I. MANNUCCl, E. et al. Comparison among different insulin sensitivity indices in obese patients. Diabet Med, v. 20, n. 6, p. 462-6, 2003

52. MANZELLA, D. et al. Blood pressure and cardiac autonomic nervous system in obese type 2 diabetic patients: effect of metformin administration. Am J Hypertens, v. 17, n. 3, p. 223-7, 2004.

53. MARUYAMA, S. et al. Serum leptin level as an indicator to predict the clinical efficacy of troglitazone in patients with type 2 diabetes mellitus. Diabetes Res Clin Pract, v. 53, n. 3, p. |6|-4, 200|.

54. MATHER, K. J. et al. Improved endothelial function with metformin in type 2 diabetes mellitus. J Am Coll Cardiol, v. 37, n. 5, p. 1344-50, 2001.

55. MATSUDA, $M$. et al. Insulin sensitivity indices obtained from oral glucose tolerance testing. Diabetes Care, v. 22, n. 9, p. 1462-70, 1999.

56. MATSUMOTO, K. et al. Glucose tolerance, insulin secretion and insulin sensitivity in nonobese and obese Japanese subjects. Diabetes Care, v. 20, n. 10, p. 1562-8, 1997.

57. MATTHEWS, D. R. et al. Control of pulsatile insulin secretion en man. Diabetologia, v. 24, p. 231-7, 1983.

58. MATTHEWS, D. R. et al. Homeostasis model assessment: insulin resistance and beta-cell function from fasting plasma glucose and insulin concentrations in man. Diabetologia, v. 28, p. 412-19, 1985.

59. NAGASAKA, S. et al. Comparison of pioglitazone and metformin efficacy using homeostasis model assessment. Diabet Med, v. 2I, n. 2, p. |36-4I, 2004.

60. NEWMAN,T.B. et al. Delineando estudos de testes médicos. In: Hulley, S. B. et al. (org.). Delineando a pesquisa clínica. Porto Alegre: Artmed, 2003. p. 203-24.

61. OLEFSKY,J. et al. Relationship between fasting plasma insulin level and resistance to insulin mediated glucose uptake in normal and diabetic subjects. Diabetes, v. 22, p. 507-13, 1973.

62. PRADHAN, A. D. et al. Insulin, proinsulin, proinsulin:insulin ratio, and the risk of developing type 2 diabetes mellitus in women. Am J Med, v. I I4, n. 6, p. 438-44, 2003.

63. RACHMANI, R. et al. The effect of acarbose on insulin resistance in obese hypertensive subjects with normal glucose tolerance: a randomized controlled study. Diabetes Obes Metab, v. 6, n. I, p. 63-8, 2004.

64. REAVEN, G. M. Role of insulin resistance in human disease. Diabetes, v. 37, p. 1595-607, 1988.

65. RESNICK, H. E. et al. Insulin resistance, the metabolic syndrome, and risk of incident cardiovascular disease in nondiabetic American Indians: the Strong Heart Study. Diabetes Care, v. 26, n. 3, p. 861-7, 2003.

66. ROSENBLATT, S. et al. The impact of pioglitazone on glycemic control and atherogenic dyslipidemia in patients with type 2 diabetes mellitus. Coron Artery Dis, v. I2, n. 5, p. 4I3-23, 200 I.

67.SABUNCU,T. et al.The effects of sibutramine and orlistat on the ultrasonographic findings, insulin resistance and liver enzyme levels in obese patients with non-alcoholic steatohepatitis [resumo]. Rom J Gastroenterol, v. I2, n. 3, p. 189-92, 2003.

68. SEKIYA, M. et al. Beneficial effect of troglitazone, an insulinsensitizing antidiabetic agent, on coronary circulation in patients with non-insulin-dependent diabetes mellitus. Jpn Circ J, v. 65, n. 6, p. 487-90, 200 I.

69. SHIBA, T. Improvement of insulin resistance by a new insulin secretagogue, nateglinide: analysis based on the homeostasis model. Diabetes Res Clin Pract, v. 62, n. 2, p. 87-94, 2003.

70. STRACZKOWSKI, M. et al.Assessment of insulin during exercise training program in obese women. Comparison of simple indices with hyperinsulinemic euglycemic clamp technique [resumo]. Pol Arch Med Wewn, v. 109, n. 5, p. 483-8, 2003.

7l. STUMVOLL, M. et al. Use of the oral glucose tolerance test to assess insulin release and insulin sensitivity. Diabetes Care, v. 23, n. 3, p. 295-30I, 2000.

72.TAN, M. et al. Effects of pioglitazone and glimepiride on glycemic control and insulin sensitivity in Mexican patients with type 2 diabetes mellitus: A multicenter, randomized, double-blind, parallel-group trial. Clin Ther, v. 26, n. 5, p. 680-93, 2004.

73.TAN, M. H. et al. Pioglitazone as monotherapy or in combination with sulfonylurea or metformin enhances insulin sensitivity (HOMA-S or QUICKI) in patients with type 2 diabetes. Curr Med Res Opin, v. 20, n. 5, p. 723-8, 2004.

74.TANIGUCHI, A. et al. Effects of bezafibrate on insulin sensitivity 
and insulin secretion in non-obese Japanese type 2 diabetic patients. Metabolism, v. 50, n. 4, p. 477-80, 2001.

75. TANIGUCHI, A. et al. The role of de body mass index and triglyceride levels in identifying insulin-sensitive and insulinresistant variants in Japanese. Metabolism, v. 49, n. 8, p. |00|-5, 2000.

76. TAVERNA, M. J. et al. Beta-cell function evaluated by HOMA as a predictor of secondary sulphonylurea failure in type 2 diabetes. Diabet Med, v. 18, n. 7, p. 584-8, 2001 .

77.TORRENS, J. I. et al. Ethnic differences in insulin sensitivity and beta-cell function in premenopausal or early perimenopausal women without diabetes. The Study of Women's Health Across the Nation (SWAN). Diabetes Care, v. 27, n. 2, p. 354-61, 2004

78. TUAN, C. Y. et al. Usefulness of plasma glucose and insulin concentrations in identifying patients with insulin resistance. Am J Cardiol, v. 92, n. 5, p. 606-10, 2003.

79. VIALETTES, B. et al. Early stages of beta-cell deficiency: the anticipated chronicle to type 2 diabetes. Diabetes Metab, v. 29, n. 2 Pt 3, p. II-5, 2003.

80. WALLACE, T. M. et al. An increase in insulin sensitivity and basal beta-cell function in diabetic subjects treated with pioglitazone in a placebo-controlled randomized study. Diabet Med, v. 21, n. 6, p. 568-76, 2004.

8I. WALLACE, T. M. et al. Use and abuse of HOMA modeling. Diabetes Care, v. 27, n. 6, p. 1487-95, 2004.
82.WOLFFENBUTTEL, B. H. et al. Prognostic factors for successful insulin therapy in subjects with type 2 diabetes. Neth J Med, v. 54, n. 2, p. 63-9, 1999.

83. YILDIZ, B. O. et al. Glucose intolerance, insulin resistance and hyperandrogenemia in first degree relatives of women with polycystic ovary syndrome.J Clin Endocrinol Metab, v. 88, n. 5, p. 203I-6, 2003.

84.YILDIZ, B.O. et al. Assessment of glucose intolerance and insulin sensitivity in polycystic ovary syndrome. Reprod Biomed Online, v. 8, n. 6, p. 649-56, 2004.

85.YOKOYAMA, H. et al. Quantitative insulin sensitivity check index and the reciprocal index of homeostasis model assessment are useful indexes of insulin resistance in type 2 diabetic patients with wide range of fasting plasma glucose. J Clin Endocrinol Metab, v. 89, n. 3, p. 1481-4, 2004.

86. YOSHINAGA, $\mathrm{H}$. et al. Heterogeneous relationship of early insulin response and fasting insulin level with development of non-insulin-dependent diabetes mellitus in non-diabetic Japanese subjects with or without obesity. Diabetes Res Clin Pract, v. 44, n. 2, p. 129-36, 1999.

87. ZHANG, C. L. et al. Effect of rosiglitazone on ovulation induction in women with polycystic ovary syndrome [resumo]. Zhonghua Fu Chan Ke Za Zhi, v. 39, n. 3, p. 173-5, 2004.

88. ZHENG, Z. et al. Effect of rosiglitazone on insulin resistance and hyperandrogenism in polycystic ovary syndrome [resumo]. Zhonghua Fu Chan Ke Za Zhi, v. 37, n. 5, p. 27I-3, 2002

\begin{tabular}{l|l} 
& Endereço para correspondência \\
\hline & Ernesto Pereira de Oliveira \\
Av. Adenil Falcão 615, Braślia \\
CEP 44062-161 - Feira de Santana-BA \\
Tel.: (75) 9132-8841 \\
e-mail: epo@ufba.br
\end{tabular}

